\title{
Intervención educativa comunitaria sobre la diabetes en el ámbito de la atención primaria
}

\author{
Ana Gladys Aráuz, ${ }^{1}$ Germana Sánchez, ${ }^{1}$ Gioconda Padilla, ${ }^{1}$ \\ Mireya Fernández, ${ }^{1}$ Marlene Roselló ${ }^{1}$ y Sonia Guzmán ${ }^{1}$
}

RESUMEN Objetivos. Ofrecer una alternativa de educación sobre la diabetes con la participación de pacientes, familiares y personal sanitario, adaptada a las condiciones locales y a las necesidades sentidas por estas personas.

Métodos. Se describe la metodología utilizada en una intervención educativa comunitaria sobre la diabetes de tipo 2, dirigida al primer nivel de atención. El estudio se realizó en el área de salud de El Guarco, Costa Rica. En una primera etapa se hizo un estudio cualitativo sobre los conocimientos y prácticas de los pacientes y del personal sanitario en relación con la prevención y el tratamiento de la diabetes y sobre la disponibilidad de alimentos en la comunidad. A partir de estos resultados, se desarrolló la metodología educativa, para lo cual se diseñaron un manual y dos procesos de capacitación sobre la diabetes, uno dirigido al personal sanitario y otro a los pacientes. Además, se desarrollaron estrategias comunitarias para dar sostenibilidad al proceso educativo.

Resultados. Se verificó que los pacientes no asocian el origen de la enfermedad con los antecedentes familiares ni el sobrepeso, que confunden los sintomas de hiperglucemia e hipoglucemia y que no hay homogeneidad en los mensajes de nutrición que reciben. Sobre la base del manual sobre diabetes, se capacitó al personal sanitario, cuyos conocimientos sobre el tratamiento y la prevención de la diabetes y sobre la metodología educativa mejoraron (promedio de $85 \%$ ). A su vez, el personal sanitario capacitó a los pacientes diabéticos de su comunidad (edad: $57,0 \pm 8,9$ años, 92\% mujeres), quienes mostraron después del curso una disminución de la glucemia, de $189 \pm 79 \mathrm{mg} / \mathrm{dL}$ a $157 \pm 48 \mathrm{mg} / \mathrm{dL}(P=0,03)$, y de la hemoglobina glucosilada, de $11,3 \pm 2,4 \%$ a $9,7 \pm 2,3 \%(P=0,05)$. No hubo cambios significativos del peso ni del perfil lipídico, a excepción de los triglicéridos, que disminuyeron $(P=0,04)$.

Conclusiones. El nivel primario de atención es ideal para ejecutar programas educativos sobre el tratamiento y la detección temprana de la diabetes dirigidos a los pacientes, sus familiares y el personal sanitario. Se logró incorporar el programa educativo a la planificación anual del área de salud.

Palabras clave

Intervención educativa, salud pública, diabetes, educación sobre la diabetes, participación comunitaria.

El reconocimiento de la diabetes de tipo 2 como una enfermedad crónica

\footnotetext{
Instituto Costarricense de Investigación y Enseñanza en Nutrición y Salud (Inciensa). Toda la correspondencia debe enviarse a Ana Gladys Aráuz, Instituto Costarricense de Investigación y Enseñanza en Nutrición y Salud (Inciensa), Tres Ríos. Apartado 4-2250. Costa Rica. Fax: (506) 279-5546. Correo electrónico: aarauz@inciensa.sa.cr
}

que afecta a millones de personas en el mundo ha motivado la búsqueda de diversos ámbitos de atención de salud, enfoques y metodologías que favorezcan un acercamiento real al problema, principalmente en relación con los conocimientos, las percepciones, las actitudes, los temores y las prácticas de los pacientes en el contexto familiar y comunal.

Por ello, para responder a las necesidades educativas y a los problemas de las comunidades, se están incorporando las teorías y modelos de las ciencias sociales a los programas de salud del primer nivel de atención, con 
el fin de hacerlos más flexibles para controlar y prevenir las enfermedades crónicas (1).

La diabetes es una enfermedad crónica que tiene un desarrollo gradual y para cuyo control son esenciales el consejo y la guía del personal sanitario, junto con la actitud de los pacientes ante la enfermedad (2).

Los procesos educativos son claves en las intervenciones preventivas en el ámbito comunal, particularmente aquellos que han evolucionado de una relación emisor-receptor a una comunicación en la que el profesional de la salud comparte sus conocimientos y el receptor pasa de una actitud pasiva a otra activa y responsable (3). La educación sobre la diabetes es importante porque permite informar, motivar y fortalecer a los afectados y a sus familiares para controlar, prevenir o retardar las complicaciones en el seno de la familia (4).

En Costa Rica, los programas de educación sobre la diabetes se han desarrollado tradicionalmente en los niveles secundario y terciario de atención y se han dirigido específicamente al paciente, sin incorporar al grupo familiar (5). Sin embargo, con la readecuación del modelo de salud, el nivel primario asume la responsabilidad de la educación y la detección temprana de los pacientes diabéticos en la comunidad. Estas acciones proporcionan una oportunidad de desarrollar estrategias educativas activas y participativas que trasciendan la visión asistencial y local hacia un enfoque más activo de la promoción de la salud y de la prevención de las enfermedades crónicas prevalentes, como la diabetes.

Este artículo describe la metodología utilizada para el desarrollo de un proyecto comunitario de intervención educativa sobre la diabetes, dirigida al primer nivel de atención, y presenta los resultados de dicha intervención. El objetivo es ofrecer una alternativa de educación con participación de los pacientes, de sus familiares y del personal sanitario, adaptada a las condiciones locales y a las necesidades percibidas por estas personas.
Las metas del proyecto de intervención fueron las siguientes:

- Estimular al personal sanitario para que desarrolle procesos educativos que involucren a la familia y a la comunidad.

- Estimular al paciente para que se implique activamente en los procesos educativos y exija atención educativa sobre la diabetes en los Equipos Básicos de Atención en Salud (EBAIS).

- Incluir el programa de educación sobre la diabetes en los planes operativos y en los compromisos de gestión del área de salud.

- Involucrar a las organizaciones comunitarias en la implantación de las actividades de prevención y detección temprana de la diabetes.

\section{MATERIALES Y MÉTODOS}

Durante 2 años, en cinco de los 10 EBAIS del área de salud de El Guarco, provincia de Cartago, Costa Rica, se desarrolló un estudio de tipo exploratorio y descriptivo que permitió diseñar una intervención educativa sobre la diabetes en el ámbito comunal. Cada EBAIS atiende a 4000 personas y está integrado por un médico general, una enfermera auxiliar y un asistente de atención primaria. El área de salud cuenta con un director y personal de apoyo en odontología, microbiología, trabajo social, enfermería y administración.

El protocolo de este proyecto fue aprobado por el Comité Ético Científico del Instituto Costarricense de Investigación y Enseñanza en Nutrición y Salud (Inciensa) y se obtuvo, por escrito, el consentimiento de cada participante. Del total de 61 personas con diabetes registradas en los EBAIS de esta área de salud, se identificaron 42 diabéticos de tipo 2 que cumplieron con los siguientes criterios de selección: vivir en la zona, asistir regularmente al control en los EBAIS, edad comprendida entre los 30 y los 60 años, y ausencia de complicaciones crónicas que les impidieran proporcionar información y asistir a las actividades del proyecto. De esos 42 pacientes, cinco fueron excluidos, uno porque no se le pudo localizar, otro porque no aceptó participar, y tres porque vivían fuera del área de cobertura.

La intervención se dividió en tres etapas (figura 1); en la práctica, la segunda y la tercera se ejecutaron simultáneamente.

\section{Primera etapa}

Se realizó un estudio cualitativo de los conocimientos y prácticas de los pacientes y del personal sanitario acerca de la prevención y tratamiento de la diabetes en el hogar, de la percepción que tiene el paciente de la gravedad y del riesgo familiar de padecer la enfermedad, así como de las motivaciones y barreras para tratar adecuadamente la diabetes. Con este fin, se seleccionaron al azar 20 de los 37 pacientes como informantes claves. Para la recolección de los datos se utilizó una entrevista no estructurada dirigida a los pacientes y un grupo focal de discusión con el personal sanitario. Los datos fueron analizados con el apoyo del programa Ethnograph V4.0. Las categorías de análisis empleadas fueron el conocimiento de la enfermedad, la percepción de la vulnerabilidad y de la gravedad, y las barreras y motivaciones de los pacientes para tratar la diabetes (Health Belief Model) (6-8)

Paralelamente al estudio cualitativo, se desarrolló un estudio de disponibilidad de alimentos en el que participaron los 37 pacientes seleccionados y los dueños de los 52 comercios de alimentos de la comunidad; mediante una entrevista estructurada, se indagó sobre el lugar de compra, los tipos de alimentos comprados, las razones para comprarlos o no y la siembra de alimentos en las huertas, patios o fincas familiares.

\section{Segunda etapa}

A partir de los resultados de los estudios anteriores, se diseñó un manual educativo dirigido al personal 
FIGURA 1. Estrategia del proyecto educativo sobre la diabetes sacarina. El Guarco, Costa Rica, 1999

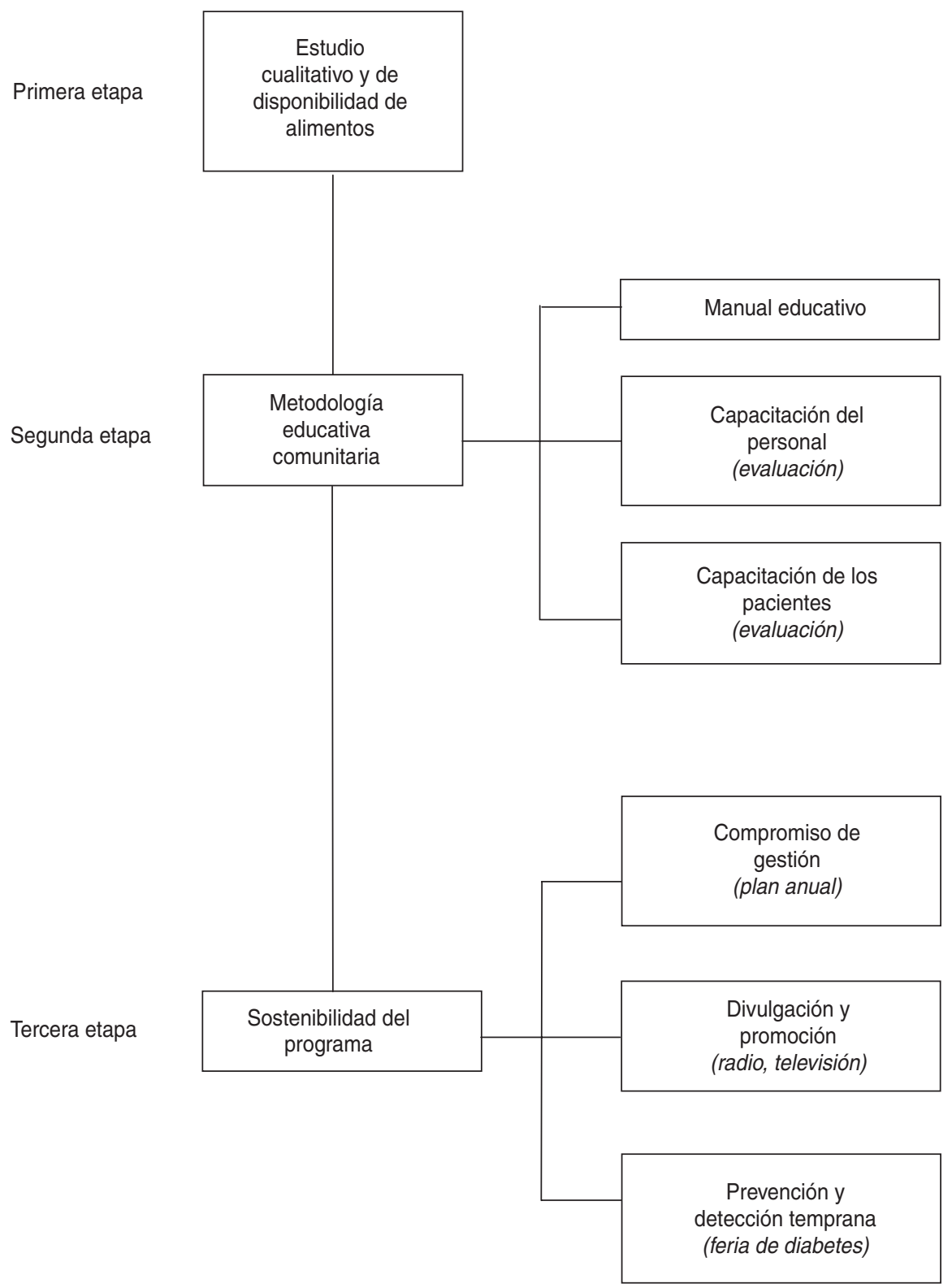

sanitario del primer nivel de atención, como herramienta didáctica para que el grupo coordinador del proyecto capacitara a dicho personal y este, a su vez, capacitara a los pacientes diabéticos. El resultado de la capacitación del personal se evaluó mediante una prueba escrita sobre aspectos tales como los conocimientos acerca de la enfermedad, la alimentación, las técnicas de enseñanza y las característi- cas de la comunidad. La capacitación de los pacientes llevada a cabo por el personal sanitario se evaluó antes y después de los 4 meses que duró el proceso de capacitación, durante los cuales se iniciaron las actividades destinadas a conseguir la sostenibilidad del programa en el área. Dicha evaluación se realizó por medio de indicadores antropométricos, bioquímicos, dietéticos y de conocimientos y percepciones sobre la enfermedad. Este artículo presenta únicamente los resultados de los indicadores bioquímicos y antropométricos.

Para el análisis de los indicadores bioquímicos se obtuvo una muestra de sangre extraída por punción venosa tras 12 horas de ayuno. La glucosa, el colesterol y los triglicéridos séricos fueron analizados con métodos enzimáticos colorimétricos (Laboratorio Weiner, Rosario, Argentina). Para determinar el colesterol de las lipoproteínas de alta densidad (HDL) se utilizó el método de precipitación con polietilenglicol $6000 \mathrm{al} 20 \%$. El colesterol de las lipoproteínas de baja densidad (LDL) se calculó con la fórmula de Friedewald (9). La glicohemoglobina presente en el lisado de glóbulos rojos se extrajo mediante una resina catiónica de intercambio débil y se determinó con un método colorímetrico no enzimático (Teco Diagnostics, Anaheim, California).

\section{Tercera etapa}

En esta fase se desarrollaron estrategias para dar sostenibilidad al proceso educativo en el área de salud, en los EBAIS y en la comunidad, además de promover la participación comunitaria en la detección temprana y prevención de la diabetes, involucrando al personal sanitario, a los pacientes, a sus familiares, a las organizaciones comunitarias y a la empresa privada.

\section{RESULTADOS}

\section{Primera etapa: estudio cualitativo y de disponibilidad de alimentos}

Se verificó que, a pesar del esfuerzo e interés del personal de los EBAIS por desarrollar los programas educativos, muchas veces carecen de los conocimientos y las herramientas adecuadas que permitan satisfacer las demandas y las necesidades educativas de la población que atienden, lo cual se refleja en los resultados resumidos en el cuadro 1. 
CUADRO 1. Principales resultados del estudio cualitativo realizado en los pacientes con diabetes de tipo 2. El Guarco, Costa Rica, 1998

Conocimientos y prácticas populares más frecuentes:

- Asocian el origen de la diabetes con el consumo de azúcares y licores y con los nervios y sufrimientos, pero no con la herencia ni el sobrepeso

- Consideran que las mujeres son más vulnerables que los hombres a padecer diabetes

- Perciben la diabetes como una enfermedad incurable y peligrosa que no se puede prevenir, aunque consideran que se pueden retrasar sus complicaciones

- A pesar de los antecedentes familiares, a la mayoría la enfermedad los tomó por sorpresa

- Confunden los síntomas de hiperglucemia e hipoglucemia con los de otras enfermedades, como la hipertensión

- Modifican el tratamiento médico (dosis y horario) y en algunos casos lo complementan con remedios caseros

- Temen el uso y consecuencias de la insulina

- Identifican varios tipos de diabetes dependiendo del tratamiento, de la edad de aparición y de la capacidad para sanar heridas

Principales barreras:

- La familia se constituye en una barrera más que en un apoyo, particularmente en el caso de las mujeres

- La comunicación entre el paciente y el personal de salud es deficiente

- No existe unidad de conceptos en los mensajes de alimentación que reciben

- La dieta recomendada es restrictiva y no tiene en cuenta los hábitos alimentarios ni la disponibilidad de alimentos en la comunidad

- Las recomendaciones dietéticas son difíciles de implantar

El estudio de disponibilidad de alimentos en los locales comerciales mostró variedad en el grupo de cereales, leguminosas y bocadillos procesados; sin embargo, a pesar de ser El Guarco una zona agrícola, el surtido de frutas, vegetales, raíces y tubérculos fue muy limitado. Esto se reflejó en la dieta doméstica, constituida fundamentalmente por arroz, frijoles y macarrones como platos básicos, cocinados con aceite o manteca vegetal, y por café con azúcar; las frutas, los vegetales, las carnes y los productos lácteos no forman parte de la dieta básica.

Los resultados anteriores se presentaron y discutieron con el personal sanitario, con el propósito de dar a conocer la problemática y las necesidades de los pacientes, además de definir los contenidos del manual educativo y el planteamiento de la estrategia en la comunidad para adaptarlos a las condiciones socioeconómicas y culturales.

\section{Segunda etapa: proceso educativo}

El proceso educativo se estructuró de manera que respondiera a las necesidades del personal sanitario, de los pacientes y del área de salud. El perso- nal sanitario identificó como prioridades la capacitación en relación con la diabetes, el uso de metodologías participativas y el establecimiento de un programa de atención educativa sobre la diabetes.

Manual educativo. La metodología para elaborar el manual se presenta en la figura 2. Se consideraron como principios básicos del proceso educativo y del manual, la integridad del individuo, sus necesidades biológicas, psicológicas y sociales, su contexto socioeconómico, cultural, familiar y comunitario, y su protagonismo en el control de la enfermedad y en la formación y promoción de hábitos saludables en la familia y en la comunidad, para prevenir la enfermedad y sus complicaciones.

Sobre la base de las necesidades de los pacientes, también se definieron y promovieron estilos de vida saludables, con alternativas viables, que permitieran a la familia, al paciente y al personal sanitario tomar decisiones acertadas, considerando la disponibilidad local de alimentos y su aceptación.

A los contenidos del manual se les dio un enfoque positivo $y$, en particular, se eliminó la dieta de los "no", dado que uno de los resultados del estudio cualitativo fue que la restricción alimentaria es un concepto institucional que aún prevalece en el tratamiento de la diabetes. Por ejemplo, anteriormente el personal sanitario recomendaba no consumir frijoles, carne, yuca, camote, pan, tortillas y frutas dulces, entre otros. En los contenidos educativos también se integró el tratamiento de otros factores de riesgo de enfermedad cardiovascular habitualmente presentes en los diabéticos, tales como dislipidemias, obesidad e hipertensión arterial.

Debido a que la alimentación fue uno de los aspectos más importantes para el personal sanitario y para los pacientes, el manual educativo dedicó gran parte de sus contenidos al desarrollo de este tema. Además, dado que en este nivel de atención se carece de recursos humanos en el campo de la nutrición, se consideró fundamental la capacitación del personal de los EBAIS.

A partir de los conocimientos y percepciones que los pacientes y sus familiares han ido elaborando sobre la diabetes, producto de diferentes fuentes de información, como los servicios de salud, los medios de comunicación y las experiencias familiares y personales con la enfermedad, se identificaron las preguntas clave para que los contenidos educativos del manual dieran respuesta a estas interrogantes. El manual consta de dos documentos: una guía para el personal sanitario y otro con el desarrollo de los temas identificados. La guía para el personal abarca:

- Generalidades sobre la estructura de las sesiones educativas, tales como la introducción, los contenidos de la sesión y las conclusiones.

- Técnicas de enseñanza participativas.

- Una guía para la redacción de los mensajes educativos.

- Actividades de evaluación, tanto del proceso, como del impacto del programa.

- Principios sobre la educación de adultos.

- El perfil del diabético al cual están dirigidos los contenidos del manual. 
FIGURA 2. Flujograma para elaborar el manual educativo

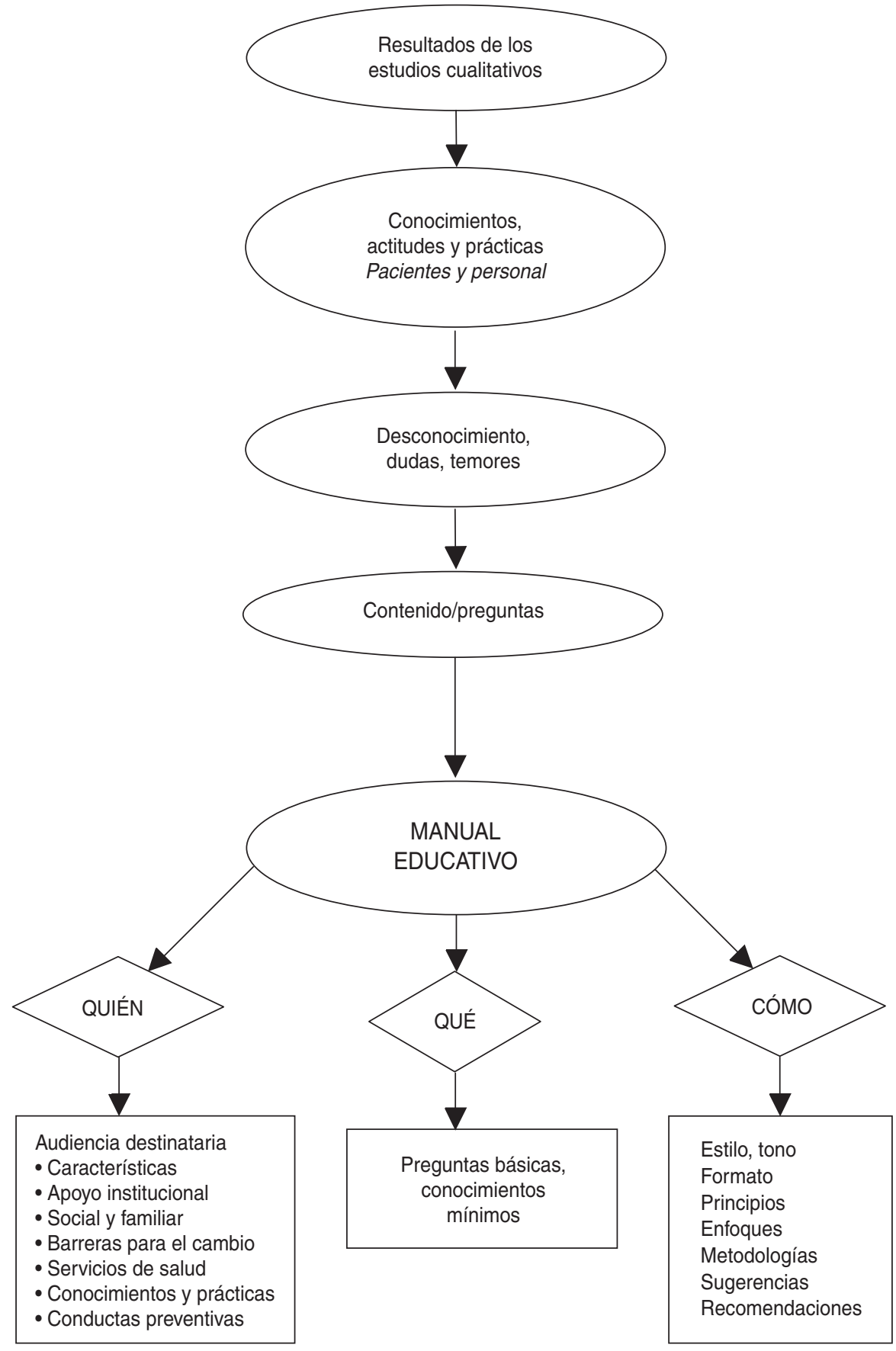

Los temas desarrollados en el manual dan respuesta a las siguientes preguntas:

- ¿Qué es la diabetes? ¿Cómo se relaciona con la alimentación?

- ¿Cuál es el tratamiento que se recomienda a las personas con diabetes de tipo 2?
- ¿Cómo prevenir las complicaciones de la diabetes?

Capacitación del personal sanitario. El manual educativo fue revisado con el personal de atención primaria y, una vez incorporadas las observaciones pertinentes, se capacitó a 25 miembros de 10 EBAIS (médicos, enfermeras y técnicos de atención primaria) y al personal de apoyo del área, tales como asistentes dentales, microbiólogos y odontólogos.

El grupo coordinador del proyecto desarrolló el proceso de capacitación del personal, que incluyó los temas del manual educativo distribuidos en ocho sesiones de dos horas cada una. Se utilizaron las técnicas participativas solicitadas previamente por el personal en los grupos focales de discusión, tales como las representaciones, las demostraciones, la discusión de casos o la elaboración de material visual, como la confección de títeres o marionetas. Durante el curso, el personal elaboró el material educativo adaptado a su contexto socioeconómico y cultural.

Con respecto a la evaluación del personal, inicialmente el $100 \%$ de los profesionales obtuvo una puntuación inferior a 80 en una escala de 100 puntos, mientras que al final, el $75 \%$ obtuvo una puntuación superior a este valor. En general hubo un aumento de los conocimientos en las áreas temáticas evaluadas, principalmente en la identificación de los alimentos que aportan azúcares de absorción lenta y rápida, así como en las técnicas didácticas participativas (cuadro 2). Llamó la atención el grado de participación y rendimiento del personal no médico, que tradicionalmente no recibe capacitación para el tratamiento de la diabetes, pero que también debe entrar a formar parte de la atención integral del paciente diabético.

- ¿Cómo distribuir los alimentos durante el día?

- ¿Cuáles son las características de una alimentación saludable para la persona con diabetes de tipo 2 y su familia?

- ¿Cuáles son los síntomas de la diabetes?
Capacitación de los pacientes. Basándose en el manual educativo, los profesionales de cuatro EBAIS desarrollaron un curso de ocho sesiones, dirigido a los 37 diabéticos identificados al inicio; no obstante por razones personales y familiares, únicamente 26 
CUADRO 2. Evolución de los conocimientos del personal sanitario tras la capacitación. El Guarco, Costa Rica, 1998

\begin{tabular}{lccc}
\hline \multicolumn{1}{c}{ Concepto } & $\begin{array}{c}\text { Inicial }^{\mathrm{a}} \\
(\%)\end{array}$ & $\begin{array}{c}\text { Final }^{\mathrm{a}} \\
(\%)\end{array}$ & $\begin{array}{c}\text { Incremento }^{\mathrm{b}} \\
(\%)\end{array}$ \\
\hline Características de la población diana & 16,0 & 35,3 & 19,3 \\
Técnicas participativas & 48,0 & 94,1 & 46,1 \\
Elaboración de mensajes educativos & 40,0 & 58,0 & 18,0 \\
Alimentación de la familia & 68,0 & 82,4 & 14,4 \\
Identificación de alimentos con azúcar de absorción rápida & 8,0 & 88,2 & 80,2 \\
Identificación de alimentos con azúcar de absorción lenta & 12,0 & 76,5 & 64,5 \\
Identificación de alimentos ricos en fibra & 40,0 & 70,6 & 30,6 \\
Alimentación adecuada en diabetes & 4,0 & 47,1 & 43,1 \\
Promedioc $^{c}$ & 57,5 & 85,3 & 27,8 \\
\hline
\end{tabular}

a \% de personas que respondieron adecuadamente.

${ }^{\mathrm{b}}$ Diferencia entre el porcentaje final y el inicial.

${ }^{c}$ Nota total de la prueba.

pacientes participaron en todo el curso y, de ellos, solo 19 asistieron a la evaluación final, ya que coincidió con festividades nacionales. Las actividades educativas se programaron por las tardes, a solicitud tanto de los pacientes como del personal sanitario, para no interferir con la consulta diaria y dedicar el tiempo necesario a la actividad educativa. En uno de los EBAIS se sumaron al proceso educativo 15 mujeres con sobrepeso $\mathrm{u}$ obesidad.

El cuadro 3 describe las características de los pacientes diabéticos que participaron en el proceso educativo. El 92\% de los participantes eran mujeres, la edad promedio del grupo fue de $57 \pm 8,9$ años (41-77), 72\% tenían diabetes diagnosticada hacía más de 5 años, $46,1 \%$ utilizaban insulina y $77 \%$ eran sedentarios (cifra que se redujo en $25 \%$ al finalizar el proceso). El cuadro 4 muestra el perfil lipídico y glucémico de los participantes. Se observó una reducción estadísticamente significativa de la glucemia en ayunas $(P=0,03)$, de la hemoglobina glucosilada $(P=0,05)$ y de los triglicéridos $(P=0,04)$.

Sostenibilidad. Como estrategia para garantizar la sostenibilidad del proceso educativo sobre la diabetes, el área de salud de El Guarco lo incorporó a su programación anual y a sus compromisos de gestión, que buscan mejorar la calidad de la atención integral al paciente diabético.
Por iniciativa del personal, se participó en el concurso "Premio a la calidad", patrocinado por la Caja Costarricense de Seguro Social (CCSS), que proporcionó una oportunidad para divulgar y compartir la experiencia educativa sobre la diabetes con las otras áreas de salud. Esta actividad tuvo reconocimiento al obtener el tercer lugar nacional.

Como parte de las actividades comunitarias de divulgación, se coordinó con la emisora local de radio para informar a la población sobre la problemática de la diabetes y sus implicaciones en la salud. Además, durante cinco meses se realizó por televisión un programa mensual de educación sobre la diabetes para comunicar los mensajes y conceptos prácticos descritos en el manual educativo.

Además, el área de salud de El Guarco realizó una "feria sobre la diabetes" (actividad comunitaria de promoción de la salud que se lleva a cabo en un lugar público, con el objetivo de realizar pruebas de detección temprana de la diabetes y de sus factores de riesgo), dirigida al personal sanitario en el "I Foro regional de mejoramiento continuo de la calidad" y organizada por la CCSS. Posteriormente, en El Guarco se organizó otra "feria sobre la diabetes" dirigida al público en general con la participación del comité local de salud, del personal sanitario de los cuatro EBAIS, de la empresa pri- vada y del grupo coordinador del proyecto. La feria se realizó en el ámbito de la Feria del Agricultor local (mercado abierto semanal de vegetales y frutas) y asistieron 172 personas adultas, a las cuales se les aplicó el formulario de factores de riesgo de la diabetes, propuesto por la ADA (American Diabetes Association, 1999). Del total, 9,3\% presentó glucemias anormales y se detectaron dos nuevos casos probables de diabetes que fueron referidos al EBAIS de su localidad para su confirmación.

\section{DISCUSIÓN}

El primer nivel de atención en salud fue propicio para lograr cambios de actitud sobre la diabetes, tanto por parte de los pacientes como de sus familiares, del personal sanitario, de los grupos de voluntarios y de la empresa privada local. La educación sobre la diabetes y su detección temprana no es un tema reciente, pero la responsabilidad asignada al primer nivel de atención $(10,11)$ ofreció la oportunidad de conocer y compartir experiencias en su contexto sociocultural con las personas que padecen la enfermedad.

Este proyecto cambió el rígido esquema educativo vertical por otro más flexible, pues fue elaborado a partir de las necesidades de capacitación y

CUADRO 3. Características de los 26 pacientes que participaron en el proceso educativo. El Guarco, Costa Rica, 1999

\begin{tabular}{lc}
\hline Edad (años) & $57,0 \pm 8,9$ \\
Tiempo de diagnóstico de diabetes & \\
$\quad$ Más de cinco años $(\%)$ & 72,0 \\
$\quad$ Menos de cinco años $(\%)$ & 28,0 \\
Peso $(\mathrm{kg})^{\mathrm{a}}$ & $69,3 \pm 10,0$ \\
Índice de masa corporal $\left(\mathrm{kg} / \mathrm{m}^{2}\right)^{\mathrm{a}}$ & $29,6 \pm 3,8$ \\
CA/CC $(\mathrm{cm})^{\mathrm{ab}}$ & $0,87 \pm 0,1$ \\
Sedentarismo (\%) & 77,0 \\
Tabaquismo (\%) & 7,7 \\
Escolaridad & 11,5 \\
$\quad$ Analfabetos (\%) & 57,7 \\
\hline Primaria incompleta (\%) & \\
\hline
\end{tabular}

a Variables expresadas como media \pm desviación estándar. ${ }^{b}$ Relación entre la circunferencia de la cintura y de la cadera. 
CUADRO 4. Perfil lipídico y glucémico de 19 pacientes al inicio y al final del proceso educativo. El Guarco, Costa Rica, 1999

\begin{tabular}{lccl}
\hline \multicolumn{1}{c}{ Variables $^{\mathrm{a}}$} & Antes & Después & $P$ \\
\hline Colesterol total (mg/dL) & $212 \pm 42$ & $209 \pm 34$ & 0,69 \\
$\mathrm{LDL}-$ colesterol (mg/dL) & $133 \pm 43$ & $136 \pm 37$ & 0,5 \\
$\mathrm{HDL}$ - colesterol (mg/dL) & $36 \pm 12$ & $35 \pm 7,5$ & 0,66 \\
Triglicéridos (mg/dL) & $214 \pm 108$ & $187 \pm 112$ & 0,04 \\
Glucemia (mg/dL) & $189 \pm 79$ & $157 \pm 48$ & 0,03 \\
Hemoglobina glucosilada (\%) & $11,3 \pm 2,4$ & $9,7 \pm 2,3$ & 0,05 \\
\hline
\end{tabular}

a Valores expresados como promedio \pm desviación estándar.

de los problemas identificados por los pacientes diabéticos y el personal sanitario, adaptándose a los recursos del área de salud. Esto motivó la participación espontánea en las actividades educativas y permitió al paciente y al personal sanitario percibir los beneficios de la educación sobre la diabetes, lo cual garantizó la participación y sostenibilidad del proceso en ese nivel de atención.

Uno de los principales retos del proyecto fue lograr la motivación del personal sanitario para que se involucrara activamente en la educación sobre la diabetes y en las actividades de promoción de la salud en la comunidad. En este sentido, el análisis de los resultados del estudio cualitativo sobre los conocimientos, las creencias y las prácticas de los pacientes en relación con su tratamiento, así como el conocimiento de la disponibilidad de alimentos en la comunidad, fueron claves para que el personal sanitario viera la educación como una herramienta fundamental para lograr el control de la glucemia. Para el personal sanitario fue importante descubrir el contraste entre la dieta de los pacientes antes de la intervención educativa y la que ellos recomiendan y comprobar que los ingresos económicos son una de las principales limitaciones de los pacientes para adquirir esos alimentos.

El apoyo familiar es indispensable para que el paciente afronte los problemas diarios de su enfermedad y para mejorar su control glucémico (12); sin embargo, muchas veces no existe tal apoyo (13); más bien, en este estudio la familia se percibió como una barrera para cumplir con el tratamiento, sobre todo para las mujeres (5), que debían adaptarse a los gustos y preferencias alimentarias de los miembros de la familia; por lo tanto el proceso educativo consideró fundamental integrar a las familias en la capacitación.

El personal de salud cumplió su papel de facilitador del proceso de comunicación y el paciente asumió el papel protagónico en su control, como se ha descrito anteriormente (14). Además, los pacientes motivados exigieron mayor atención educativa del personal, lo cual los llevó a preparar materiales y mensajes educativos adaptados a la situación.

El logro de este proyecto, a diferencia de otros procesos educativos cortos exitosos $(15,16)$, fue la labor educativa directa del personal capacitado de atención primaria. La reducción significativa de los valores séricos de la hemoglobina glucosilada fue un indicador del éxito del proceso. Se ha demostrado que su disminución en un 1 a $2 \%$ contribuye a reducir las complicaciones microvasculares en un 29 a 35\% (17).

En general, aunque no se observó una reducción del peso corporal, hubo beneficio sobre el control de la glucemia (18). Se debe considerar que estos pacientes presentaron las características descritas de la resistencia a la insulina (19), 40\% utilizó insulina y eran sedentarios, lo que dificulta la reducción de peso. Estas mismas limitaciones fueron descritas en otros estudios en la población diabética latinoameri- cana, donde la prevalencia de antecedentes familiares es alta $(20,21)$. Otra limitación para la reducción de peso fue la presencia de glucemias elevadas, mantenidas durante varios años, y la misma evolución de la enfermedad. En un estudio realizado en una población semejante se han descrito datos similares (20) y la primera modificación de sus hábitos alimentarios que realizaron los pacientes capacitados fue el cambio del tipo de alimentos y la regulación del horario, sin un cambio sustancial en la cantidad de alimentos energéticos consumidos (22); esta situación pudo haber contribuido a que la glucemia postprandial regresara a niveles preprandiales antes de la siguiente comida y se mejorara el control de la glucemia (23) sin que el peso presentara cambios importantes. La oportunidad y el efecto de un programa educativo semejante a este sobre el control de la diabetes y el peso corporal será mayor en los pacientes recién diagnosticados y que aún no han desarrollado complicaciones crónicas.

Las metas del proyecto fueron cumplidas. En la etapa de sostenibilidad, las iniciativas para participar en los concursos regionales y nacionales para promocionar este programa educativo y la incorporación del comité de salud local a las actividades sobre la diabetes fue el resultado de la motivación del personal sanitario. Para la ejecución de los programas comunitarios en este nivel de atención, la clave fue la motivación del director del área de salud y su equipo.

A sugerencia del personal de dos EBAIS, las participantes en el programa de control de peso se sumaron al curso sobre diabetes, dando como resultado un grupo de 40 personas. Esta situación interfirió con la propuesta original de hacer grupos pequeños para facilitar la interacción entre el personal y los pacientes, pero fue una oportunidad para que se motivaran y modificaran sus hábitos alimentarios y de actividad física, ya que casi todas las mujeres con sobrepeso tenían familiares cercanos con diabetes. Además, el mismo sobrepeso constituye un factor de riesgo de padecer la 
enfermedad, concepto que anteriormente desconocían.

En conclusión, esta experiencia permite afirmar que la atención primaria es ideal para ejecutar programas educativos, tanto de tratamiento como de prevención y detección temprana de la diabetes; en este nivel de atención hay entre los pacientes, sus familiares, la comunidad y el personal sanitario una relación de familiaridad que favorece el apoyo grupal y el intercambio de experiencias dentro del mismo contexto socioeconómico y cultural. Si los programas educativos están adaptados al personal y a los pacientes de este nivel, el beneficio del mejoramiento de la calidad de la atención lo recibe directamente el paciente, al controlar su glucemia y disminuir las complicaciones a corto y largo plazo, con la consiguiente mejora de su calidad de vida (24). Por otra parte, la integración del grupo familiar en los programas educativos brinda apoyo al paciente y contribuye a concienciar a la familia del riesgo de padecer diabetes, lo cual facilita las acciones preventivas y de diagnóstico temprano de la enfermedad en la comunidad (25).

\section{REFERENCIAS}

1. La aplicación de teorías y técnicas de las ciencias sociales a la promoción de la salud. Rev Panam Salud Publica 1998;4:142-148.

2. Hiss R. The activated patient: a force for change in diabetes health care and education. Diabetes Educ 1986;12(Suppl): 225-231.

3. Manual de educación sanitaria para equipos de atención primaria. Diabetes Mellitus tipo 2. Novo Nordisk Pharma; 1995.

4. García R, Suáres R. La educación en diabetes en Cuba, retrospectiva de las tres últimas décadas. Rev Cub Endocrinol 1999;10(Suplemento):9-12.

5. Morice A, Roselló M, Aráuz AG, Sánchez G, Padilla G. Diabetes mellitus en Costa Rica: un análisis interdisciplinario. Tres Ríos, Costa Rica: Inciensa; 1999.

6. Baranowski T. Methodologic issues in selfreport of health behavior. J Sch Health 1985; 55:179-182.

7. Simons J. Components of cultural variation in the mother's contribution to child survival. London: London School of Hygiene and Tropical Medicine, University of London, Centre for Population Studies; 1989.

8. Baranowski T. Beliefs as motivational influences at stages in behaviour change. International Quarterly of Community Health Education 1992;13:3-29.

9. Frieldwald WT, Levy RI, Fredickson DS. Estimation of the concentration of low-density lipoprotein cholesterol in plasma, without the use of the preparative centrifuge. Clin Chem 1972;18:499-502.
Agradecimiento. El equipo de investigadoras expresa su reconocimiento a los pacientes diabéticos por su participación y valiosa información, al personal de salud de la comunidad de El Guarco, especialmente a los doctores Alejandro Ramírez y Mylena Quijano, por su apoyo logístico, a las doctoras Lowella Cunninghan y Sarita Rodríguez del Inciensa, responsables de los análisis de laboratorio, y al doctor Eric Mora Morales. Nuestro agradecimiento también al Inciensa-Desaf y a la Organización Panamericana de la Salud por el financiamiento del proyecto.
10. Alleyne G. La diabetes: una declaración para las Américas. Bol Oficina Sanit Panam 1996; 121:461-466.

11. Llanos G, Libman I. La diabetes en las Américas. Bol Oficina Sanit Panam 1995;118:1-17.

12. Rodríguez-Morán M, Guerrero-Romero JF. Importancia del apoyo familiar en el control de la glucemia. Salud Publica Mex 1997;39: 44-47.

13. Valdez-Figueroa IA, Aldrete-Rodríguez MG Alfaro-Alfaro N. Influencia de la familia en el control metabólico del paciente diabético tipo II. Salud Publica Mex 1993;35:464-470.

14. García R, Suárez R, Mateo-de-Acosta O. Comunicación y educación interactiva en salud y su aplicación al control del paciente diabético. Rev Panam Salud Publica 1997;2:32-36.

15. Puente GG, Salinas AM, Villareal RE, Garza ME, Albarrán GT, Elizondo GR. Estrategia educativa para el control del paciente diabético tipo 2. Revista de Enfermería 1999;7:93-98.

16. Lascano BG, Rodríguez MM, Guerrero RF. Eficacia de la educación en el control de la glucemia de pacientes con diabetes tipo 2 . Revista Médica del Instituto Mexicano del Seguro Social 1999;37:39-44.

17. Klein R, Klein BE, Moss SE. Relation of glycemic control to diabetic microvascular complications in diabetes mellitus. Ann Intern Med 1996;124;90-96.

18. Wing RR, Koeste R, Epstein LH, Nowalk MP, Gooding W, Becker D. Long-term effect of modest weight loss in type II diabetic patients. Arch Intern Med 1987;147:1749-1753.
19. Henry RR. Glucose control and insulin resistance in non-insulin-dependent diabetes mellitus. Ann Inten Med 1996;124:97-103.

20. Rodríguez $\mathrm{O}$, Aráuz AG, Roselló M, Jiménez M, Vargas M. Efecto de un modelo educativo en diabetes mellitus sobre el control metabólico. Revista de la Asociación Latinoamericana de Diabetes 1998;6:205-211.

21. Rodríguez MM, Guerrero JF. Estudio comparativo de pacientes con diabetes tipo 2 , habitantes de comunidades urbanas y rurales. Revista Médica del Instituto Mexicano del Seguro Social 1998;36:191-197.

22. Aráuz AG, Roselló M, Padilla G, Rodríguez O, Jiménez M, Guzmán S. Modificación de prácticas alimentarias en diabéticos no insulino dependientes: efecto de una intervención educativa multidisciplinaria. Rev Costarric Cienc Med 1997;18:15-29.

23. Rost K, Schmidt L. Can health education programs improve the metabolic control of NIDDM patients?. Prog Diabetes 1992;3:1-6.

24. American Diabetes Association. Standards of medical care for patients with diabetes mellitus. Diabetes Care 1999;22(Suppl 1): S32-S41.

25. American Diabetes Association. Screening for type 2 diabetes. Diabetes Care 1999;22 (Suppl 1):S20-S23.

Manuscrito recibido el 13 de julio de 2000. Aceptado para publicación, tras revisión, el 12 de febrero de 2001. 
ABSTRACT Objective. To offer an educational alternative on diabetes, with the participation of patients with type 2 diabetes, their family members, and health care providers, adapted to local conditions and to these persons' felt needs.

\section{A community diabetes educational intervention at the primary-care level}

Methods. Focused on the primary-care level, this program was carried out in the health area of El Guarco, which is in the province of Cartago, Costa Rica. The first stage of the project included a qualitative study of the knowledge and practices of both patients and health care providers, looking at diabetes prevention and control and the local availability of foods. Based on those results, an educational methodology was developed, educational manuals were written, and courses for health care providers, patients, and patients' families were implemented. Other strategies were developed to make the effort sustainable.

Results. We found that patients did not associate family history or obesity with diabetes and that those persons were also confused about the symptoms of diabetes. Patients also received inconsistent nutrition messages from health care providers. Using the diabetes education manual as a base, the providers increased their knowledge of diabetes prevention, treatment, and education by an average of $85 \%$. The diabetic patients who received educational training (mean age, 57.0 years, with a standard deviation of 8.9 years; $92 \%$ women) improved their glycemic control. Blood glucose levels decreased from $189 \pm 79 \mathrm{mg} / \mathrm{dL}$ (average and standard deviation) to $157 \pm 48 \mathrm{mg} / \mathrm{dL}$ $(P<0.05)$, and glycosylated hemoglobin (HbA1c) went from $11.3 \% \pm 2.4 \%$ to $9.7 \% \pm$ $2.3 \%(P=0.05)$. There were no significant changes in body weight or lipid profile, except for triglycerides, which declined $(P<0.05)$.

Conclusions. This educational program was successfully incorporated into the regular activities of the El Guarco-area health centers. The primary-care level is ideal for carrying out educational programs for diabetes treatment and early detection that are directed at patients, their families, and health care providers.

\section{Premio Fred L. Soper en Salud Interamericana, 2001}

\section{Fecha límite: 31 de julio de 2001}

La Fundación Panamericana de la Salud y Educación (PAHEF) solicita candidaturas para el premio del año 2001 en honor del doctor Fred L. Soper, quien fuera Director de la OSP de 1947 a 1959. Además de los servicios prestados a la OPS, el doctor Soper desempeñó un importante papel en la lucha contra la fiebre amarilla y otras enfermedades infecciosas en el Brasil, como parte de su trabajo con la Fundación Rockefeller en los años treinta y cuarenta, así como en la lucha contra el tifo en el África septentrional e Italia durante la segunda guerra mundial. El doctor Soper fue una de las figuras más destacadas del siglo en el campo de la salud interamericana.

Este premio, que consiste en un diploma y US\$ 2500 , se concede cada año a los autores de un trabajo científico publicado que aporte nueva información al campo de la salud pública, con hincapié en América Latina y el Caribe. Puede ser un informe basado en el análisis de nuevos datos, obtenidos mediante estudios experimentales o de observación, o bien un nuevo análisis de datos que ya existen. Se concede prioridad a los estudios que abarcan más de una disciplina y a los trabajos relacionados con las enfermedades infecciosas, uno de los principales campos de interés del doctor Soper durante toda su vida.

Solo pueden participar en el concurso los trabajos publicados en revistas científicas que figuran en el Index Medicus. Además, este premio solo se concede a autores vinculados con instituciones docentes, de investigación o de servicio ubicadas en países de América Latina y el Caribe (incluidos los Centros de la OPS).

Pueden concursar trabajos presentados por sus autores o en nombre de ello, siempre que se hayan publicado en el año 2000; todos los trabajos presentados a concurso tienen que haberse recibido a más tardar el 31 de julio de 2001 :

\section{Dirección para el envío de candidaturas:}

Premio Fred L. Soper

Fundación Panamericana de la Salud y Educación 525 Twenty-third Street, NW

Washington, DC 20037 - Estados Unidos email: pahef@paho.org

Información adicional por Internet: http://www.paho.org/English/PAHEF/awards.htm 